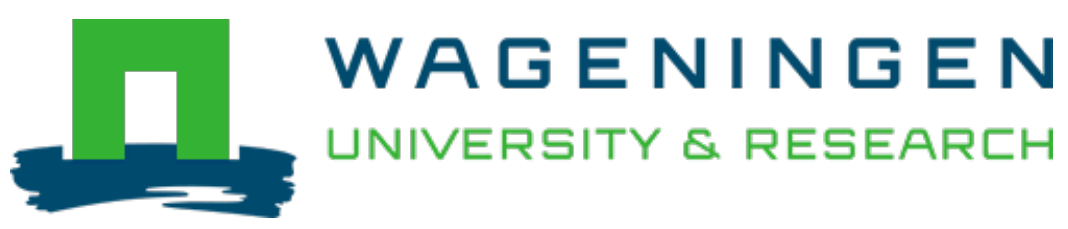

\title{
Analyzing subcellular reorganization during early Arabidopsis embryogenesis using fluorescent markers
}

\author{
Plant Embryogenesis \\ Liao, Che Yang; Weijers, Dolf \\ https://doi.org/10.1007/978-1-0716-0342-0 5
}

This publication is made publicly available in the institutional repository of Wageningen University and Research, under the terms of article $25 \mathrm{fa}$ of the Dutch Copyright Act, also known as the Amendment Taverne. This has been done with explicit consent by the author.

Article 25 fa states that the author of a short scientific work funded either wholly or partially by Dutch public funds is entitled to make that work publicly available for no consideration following a reasonable period of time after the work was first published, provided that clear reference is made to the source of the first publication of the work.

This publication is distributed under The Association of Universities in the Netherlands (VSNU) 'Article $25 \mathrm{fa}$ implementation' project. In this project research outputs of researchers employed by Dutch Universities that comply with the legal requirements of Article $25 \mathrm{fa}$ of the Dutch Copyright Act are distributed online and free of cost or other barriers in institutional repositories. Research outputs are distributed six months after their first online publication in the original published version and with proper attribution to the source of the original publication.

You are permitted to download and use the publication for personal purposes. All rights remain with the author(s) and / or copyright owner(s) of this work. Any use of the publication or parts of it other than authorised under article $25 \mathrm{fa}$ of the Dutch Copyright act is prohibited. Wageningen University \& Research and the author(s) of this publication shall not be held responsible or liable for any damages resulting from your (re)use of this publication.

For questions regarding the public availability of this publication please contact openscience.library@wur.nl 


\title{
Analyzing Subcellular Reorganization During Early Arabidopsis Embryogenesis Using Fluorescent Markers \\ Che-Yang Liao and Dolf Weijers
}

\begin{abstract}
Virtually all growth, developmental, physiological, and defense responses in plants are accompanied by reorganization of subcellular structures to enable altered cellular growth, differentiation or function. Visualizing cellular reorganization is therefore critical to understand plant biology at the cellular scale. Fluorescently labeled markers for organelles, or for cellular components are widely used in combination with confocal microscopy to visualize cellular reorganization. Early during plant embryogenesis, the precursors for all major tissues of the seedling are established, and in Arabidopsis, this entails a set of nearly invariant switches in cell division orientation and directional cell expansion. Given that these cellular reorganization events are genetically regulated and coupled to formative events in plant development, they offer a good model to understand the genetic control of cellular reorganization in plant development. Until recently, it has been challenging to visualize subcellular structures in the early Arabidopsis embryo for two reasons: embryos are deeply embedded in seed coat and fruit, and in addition, no dedicated fluorescent markers, expressed in the embryo, were available. We recently established both an imaging approach and a set of markers for the early Arabidopsis embryo. Here, we describe a detailed protocol to use these new tools in imaging cellular reorganization.
\end{abstract}

Key words Arabidopsis thaliana, Embryogenesis, Subcellular structure, Fluorescent protein, Confocal microscopy

1 Introduction

The process of embryogenesis of the flowering plant Arabidopsis thaliana provides an ideal model for addressing the fundamental question of how a single cell can give rise to a multicellular organism consisting of various cell types arranged in a precise pattern [1]. The connection between cell identity and gene activity has been the main focus of studies focusing on mechanisms underlying pattern formation during Arabidopsis early embryogenesis. As a consequence, essential techniques for analyzing cellular pattern and gene activity have been established [2-4]. However, the subcellular reorganization during oriented cell division and cell type specification, and their connection to the genetically controlled 
pattern formation process during early embryogenesis, remains to be discovered.

While genetic tools and imaging methods to dissect cell biology underlying various developmental processes have been established, three challenges obstruct the direct examination of subcellular events during embryogenesis. Firstly, most established fluorescent-protein based reporters labeling specific cellular structures are driven by constitutive promoters that have either no (e.g., CaMV 35S promoter $[5,6]$;) or weak (e.g. pUBQ10 [7, 8];) activity during early embryogenesis. Secondly, the embryo is encapsulated in the seed and fruit. While seed culture and live imaging of gene activity and cytoskeleton reorganization in zygote have been established $[9,10]$, imaging subcellular structures in more developed embryos drastically suffers from the refraction and scattering resulting from the development of the seed coat and endosperm (unpublished observation). Therefore, embryo extraction is necessary for imaging subcellular structures in embryos. A third challenge thus follows: while techniques for embryo extraction and cellular profile counterstaining for analysis of gene activity were well established [2, 3], they suffer from low embryo extraction efficiency and are not meant to preserve delicate subcellular structures, such as microtubule and actin cytoskeletons. To overcome these challenges, we recently developed and reported [11] a set of fluorescent protein reporters labeling cellular structures, and driven by an embryo-specific promoter. In addition, we optimized imaging procedures that aimed to preserve cellular structures [11]. In this chapter, we describe the detailed procedure including seed extraction, embryo isolation, microscopy specimen preparation, and key points on confocal microscope setting aim to preserve and document subcellular reorganization during early plant embryogenesis.

\section{Materials}

\subsection{Tools}

1. Forceps for collecting silique. Working surface of the tip should be less than $1 \mathrm{~mm}$ wide and flat to collect silique.

2. $92 \mathrm{~mm}$ diameter petri dish with double-sided tape. Divide the surface of the double-sided tape into a $15 \times 10 \mathrm{~mm}$ grid (Fig. la).

3. Double edged blade for safety razor. We use the ones for shaving, not industrial use (see Note 1). Preferably without grease. With the blade still in its paper sheath, bend the blade longitudinally in the middle several times to snap it in two.

4. Stereo microscope, $10 \times-40 \times$ magnification, with bottom illumination. 


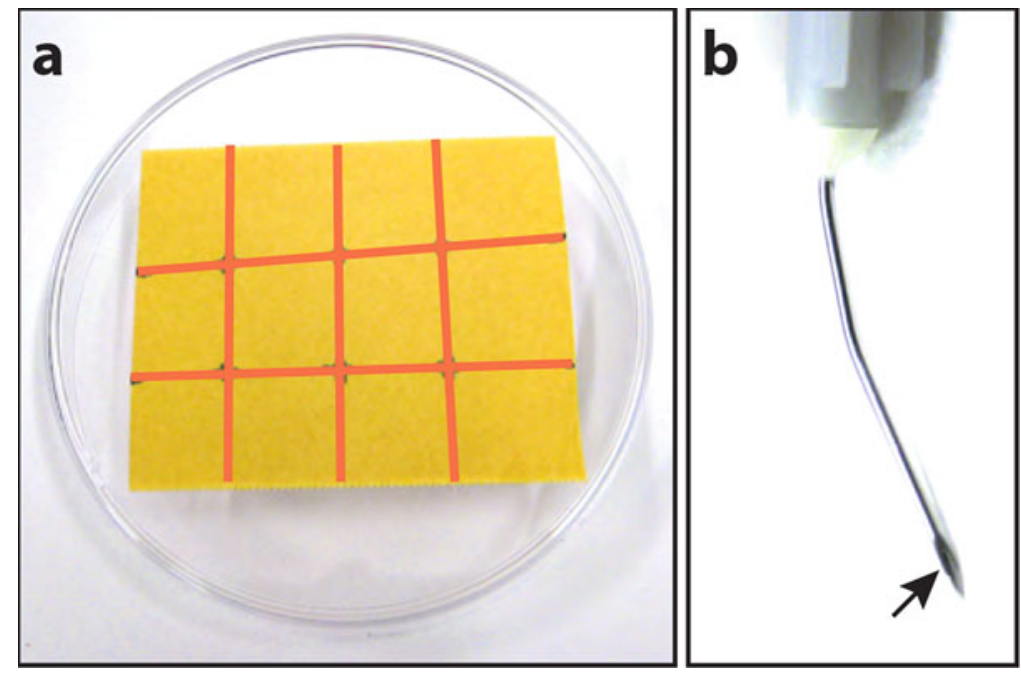

Fig. 1 Modified tools for seed extraction. (a) Petri dish with adhesive doublesided tape. The cutting pattern for the cover of the double-sided tape is marked in red. (b) Needle is bent toward the reader; note that the opening of the needle (arrow) is also toward the reader

5. $0.4 \times 15 \mathrm{~mm}$ needle mounted (by screw) on a $1 \mathrm{~mL}$ syringe. Bend the needle as in Fig. 1b, note the orientation of the needle (see Note 2 ).

6. For collecting seeds containing 1 -cell to globular stage embryos: Jeweler's forceps, straight tip with smooth working surface, tip dimension: $0.1 \times 0.06 \mathrm{~mm}$ or smaller $($ see Note 3 ).

7. For collecting seeds containing globular to torpedo stage embryos: $0.6 \times 15 \mathrm{~mm}$ needle screw mounted on $1 \mathrm{~mL}$ syringe. Dip the tip of the needle in superglue to seal the channel of the needle. Remove excessive glue with a utility knife or sand paper after the glue is dried.

$8.92 \mathrm{~mm}$ diameter petri dish lid.

9. $200 \mu \mathrm{L}$ pipette tip.

10. P100 pipette.

11. Timer.

12. Permanent marker, fine.

13. Coverslips, $24 \times 50 \mathrm{~mm}$.

14. Microscopy slide.

15. Tissues or paper towel.

16. Surgical glove.

17. Confocal microscope. Upright confocal microscope with UV $(405 \mathrm{~nm})$ for SR2200 and other light source for the fluorescent protein of choice. 
Prepare all solutions with demineralized water, if not specified otherwise.

1. $20 \%$ glucose solution: dissolve 20 g glucose in $100 \mathrm{~mL}$ demineralized water. Autoclave. Make $500 \mu \mathrm{L}$ aliquots in microcentrifuge tubes stored in $-20{ }^{\circ} \mathrm{C}$.

2. $400 \mathrm{mM}$ PIPES (piperazine- $N, N^{\prime}$-bis(2-ethanesulfonic acid)) stock solution: add $24.2 \mathrm{~g}$ PIPES with $60 \mathrm{~mL}$ demineralized water while adjusting $\mathrm{pH}$ to 6.8 with $10 \mathrm{~N} \mathrm{NaOH}$. Add demineralized water to final volume of $100 \mathrm{~mL}$. Autoclave and store at room temperature.

3. $100 \mathrm{mM}$ EGTA (ethylene glycol-bis( $\beta$-aminoethyl ether)- $N, N$, $N^{\prime}, N^{\prime}$-tetraacetic acid): dissolve $3.8 \mathrm{~g}$ EGTA with $60 \mathrm{~mL}$ demineralized water while adjusting $\mathrm{pH}$ to 7 with $10 \mathrm{~N} \mathrm{NaOH}$. Add demineralized water to final volume of $100 \mathrm{~mL}$. Store at room temperature.

4. $40 \mathrm{mM} \mathrm{MgCl}$ : dissolve $0.812 \mathrm{~g} \mathrm{MgCl}_{2} \cdot 6 \mathrm{H}_{2} \mathrm{O}$ with $90 \mathrm{~mL}$ demineralized water and add demineralized water to final volume of $100 \mathrm{~mL}$. Autoclave and store at room temperature.

5. $2 \times$ microtubule stabilizing buffer $(2 \times$ MTSB $): 200 \mathrm{mM}$ PIPES, $10 \mathrm{mM}$ EGTA, $4 \mathrm{mM} \mathrm{MgCl} 2$. Diluted with demineralized water. Make $500 \mu \mathrm{L}$ aliquot in microcentrifuge tubes and store at $-20{ }^{\circ} \mathrm{C}$.

6. 10\% SR2200 (SCRI Renaissance 2200): Mix $50 \mu \mathrm{L}$ of SR2200 with $500 \mu \mathrm{L}$ demineralized water. Make $20 \mu \mathrm{L}$ aliquot in microcentrifuge tubes and store at $-20{ }^{\circ} \mathrm{C}$.

7. $10 \mathrm{mM}$ Taxol (Paclitaxel): Dissolve in 100\% DMSO (dimethyl sulfoxide) (see Note 4 ). Make $20 \mu \mathrm{L}$ aliquot in microcentrifuge tubes and store at $-20{ }^{\circ} \mathrm{C}$.

8. Embryo general mounting solution (EGM): 10\% glucose solution.

9. Embryo general counterstaining solution (EGC): $10 \%$ glucose $+0.1 \%$ SR 2200 .

10. Embryo microtubule mounting solution (EMTM): $10 \%$ glucose, $10 \mu \mathrm{M}$ Taxol in $1 \times$ MTSB.

11. Embryo microtubule counterstaining solution (EMTC): 10\% glucose, $10 \mu \mathrm{M}$ Taxol, $0.1 \%$ SR2200 in $1 \times$ MTSB.

\section{Methods}

Every operation described below is from the perspective of a righthanded operator. 


\subsection{Transferring Seeds from the Silique to the Microscope Slide}

1. Water the plant the day before imaging ( see Note 5).

2. One will need stable hands, so avoid any activity that could increase heart rate or strain the arms prior to sample preparation.

3. With the forceps (not jeweler's), place the silique on the double-sided adhesive tape with the replum facing upward and the stigma pointing toward the operator (Fig. 2a). Place the stigma on the double-sided tape and secure it with a finger gently and "pull" the forceps gently to gently "stretch" the silique before placing the pedicel end on the double-sided tape (see Note 6).

4. Tap the silique gently along the axis of the replum with the index finger. This gives the silique firm contact with the double-sided tape to secure its position.

5. Place the petri dish under a stereomicroscope with the stigma pointing toward the operator. Following steps will be described from the perspective via stereomicroscope with 10x magnification. The prime directive is to maintain the intact structure of the seed. Embryos can hardly be extracted from damaged seed.

6. Switch on the bottom illumination of the stereomicroscope (see Note 7).

7. Secure the safety razor blade between the thumb and middle finger with index finger on the blade for fine control (Fig. 2b), cut through both ends of the silique (Fig. 2c, cut \#1 and \#2) (see Note 8).

8. From the pedicel to the stigma, cut through only one side of the right valve as close to the replum as possible with the razor blade (Fig. 2c, cut \#3) via bending the thumb and index finger (see Note 9).

9. Hold the $0.4 \times 15 \mathrm{~mm}$ needle like a pen with the point of the needle pointing downward perpendicular to the valves, opening the right valve by poking the point of the needle into opening made from cut \#3 and push the valve to the right with the thumb. Secure the valve on the double-sided tape through continuing moving the needle to the right side. The geometry of the valve will make itself against the double-sided tape providing additional stability for the following operations (Fig. 2d).

10. Rotate the petri dish so the stigma is pointing away from the operator, repeat Subheading 3.1, steps 8 and 9 to open another valve (Fig. 2e).

11. Rotate the petri dish so the stigma is pointing toward from the operator. 


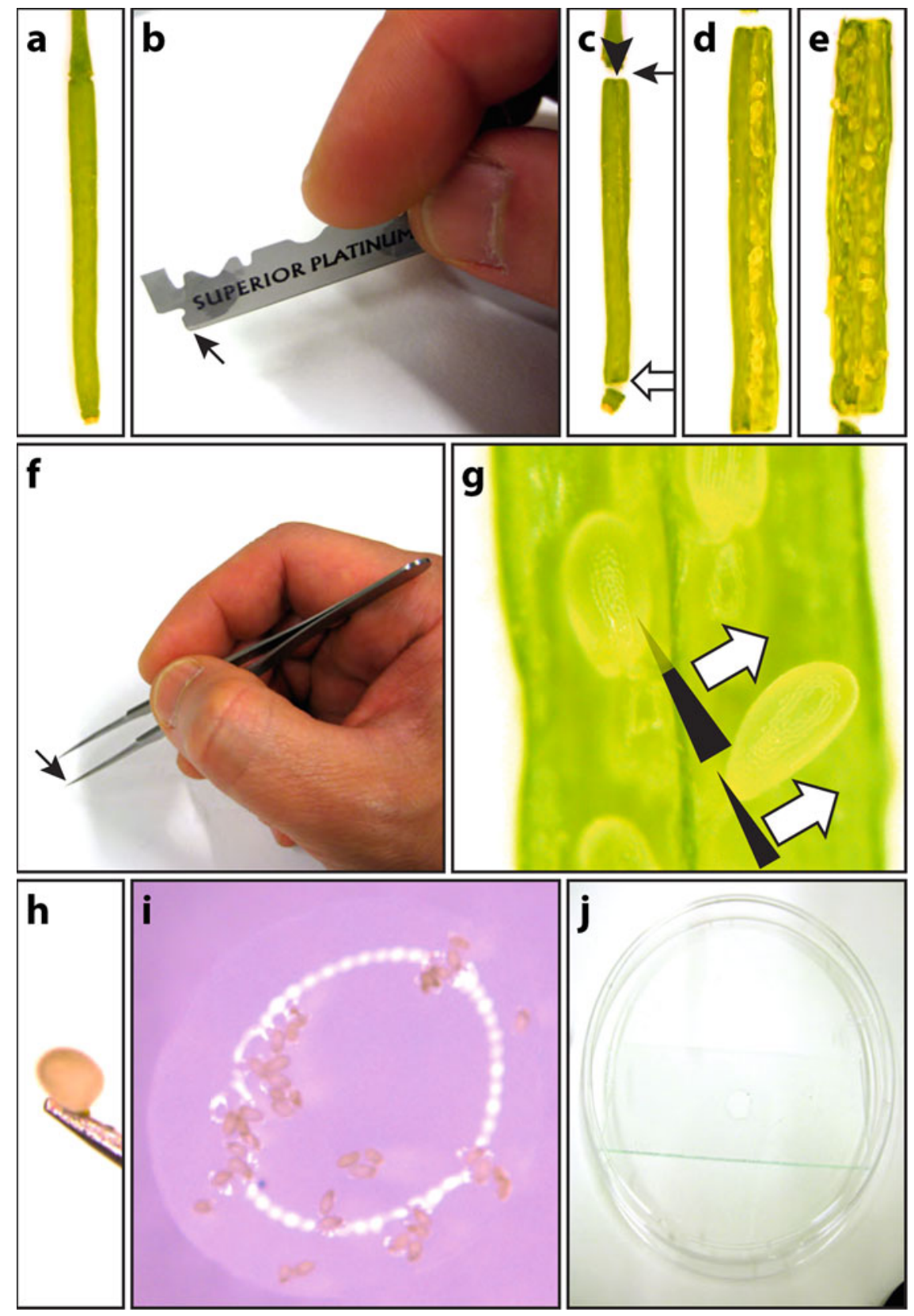

Fig. 2 Seed extraction. (a) The silique secured on the adhesive double-sided tape with its replum facing toward the reader. (b) Holding the shaving razor blade. The arrow indicates the point of contact of the razor blade with the valve. (c) Cuts to open the valve. The arrow, open arrow, and arrowhead indicate cut \#1, cut \#2, and cut \#3, respectively. (d) Silique with its right valve opened. (e) Silique with both valves opened. (f) Holding the jeweler's forceps. The arrow indicates the area of working surface contacts the seed. (g) Positions of the working surface of the jeweler's forceps during seed extraction. The black triangles indicate the working surface when extracting seed in the right or left valve. Note when extracting the seeds in the left valve, the point of the working surface is under the seed. The open arrows indicate the directions of the movement of the jeweler's forceps. (h) Extracted seed on the working surface of the jeweler's forceps. (i) Extracted seeds in the mounting medium. Note all seeds only aggregate at the same plane as the result of transferring them one-byone. (j) Petri dish covering the whole specimen to minimize mounting medium evaporation 
12. Take a microscope slide and place a drop of the mounting medium in the following volume corresponding to the seeds' developmental stage: $2-16$ cell, $10 \mu \mathrm{L} ; 16$-early heart stage, $15 \mu \mathrm{L}$; Heart to torpedo $20 \mu \mathrm{L}$ (see Note 10). Keep the medium in a shape of a droplet.

13. Place the microscope slide with the mounting medium droplet where it can be easily accessible and well lit when working with the stereomicroscope but wouldn't be disturbed during the operation. The well-lit surface of the droplet makes it easier to transfer the extracted seeds to the droplet.

14. Back to the stereomicroscope. Hold the jeweler's forceps like a pen with one working surface facing away from the operator (Fig. 2f).

15. Transfer each seed in the right valve one-by-one to the mounting medium. Start from the right valve first, from the stigma toward the pedicel (see Note 11). Place forceps' tip between the septum and the seed, gently move the tip downward to cut the funiculus, then with the working surface of the forceps against the seed, move the working surface with the seed outward (toward 2 o'clock direction) to disconnect the seed from the septum (Fig. 2g). Do not move the seed outside of the valve because once the seed contacts with the double-sided tape, any attempt to lift it from the double-sided tape is likely to damage the seed.

16. Transfer the seeds on the forceps tip (Fig. 2h) to the mounting medium on the slide by touching the seeds to the surface of the droplet (see Note 12). Once the seed is transferred to the droplet (Fig. 2i), carefully wipe tip of the forceps' tip with the tissue/paper towel to ensure a dry working surface.

17. Transfer each seed in the left valve one-by-one, from the stigma toward the pedicel, to the mounting medium. Place forceps' tip under the funiculus between the septum and the seed; gently move the tip to the right. The septum will be folded to the right with the forceps now on the funiculus (Fig. 2h). Gently move the tip downward to cut the funiculus, then with the working surface of the forceps against the seed, move the working surface with the seed outward (toward 2 o'clock direction) to disconnect the seed from the septum.

18. Transfer the seed to the mounting medium as described in Subheading 3.1, step 16.

19. Repeat Subheading 3.1, steps 17 and $\mathbf{1 8}$ until all seeds are transferred.

20. Add mounting medium over the first mounting medium droplet counting seeds in the following volume corresponding to 


\subsection{Embryo \\ Extraction}

the seeds' developmental stage: $2-16$ cells, $5 \mu \mathrm{L}$; 16-early heart stage, $7.5 \mu \mathrm{L}$; heart to torpedo $10 \mu \mathrm{L}$ (see Note 13).

21. Cover the slide with the petri dish lid (Fig. 2j) and incubate the seed in the mounting medium for $15 \mathrm{~min}$ (see Note 14).

1. At the left side of the mounting medium containing the extracted seeds, $\sim 5 \mathrm{~mm}$ apart (Fig. 3a), add staining medium in the following volume corresponding to the seeds' developmental stage: $2-16$ cell, $15 \mu \mathrm{L}$; 16-early heart stage, $22.5 \mu \mathrm{L}$; Heart to torpedo $30 \mu \mathrm{L}$ ( see Note 15).

2. Put on a surgical glove on the left hand if Taxol is used (see Note 16).

3. With the forceps (not jeweler's) in the right hand, hold the coverslip with the forceps and position the left edge of coverslip, secured by the left hand, on the left side of the droplet of the staining medium with the angle between the coverslip and the slide at $45^{\circ}$ (Fig. 3a).

4. Open the forceps but keep contact with the coverslip to support the coverslip with the tip of the forceps.

5. Lower the forceps slowly to close the coverslip without introducing air bubbles as in (Fig. 3a) (see Note 17).

6. Place the slide under the stereomicroscope. Switch on the bottom illumination.

7. Secure the slide with left thumb and middle finger and secure the coverslip with the left index finger a shown in (Fig. 3c) (see Note 18 ).

8. Start the timer, the specimen can be used for imaging for an hour from now on.

9. Secure a $200 \mu \mathrm{L}$ pipette tip in the right hand with the thumb, index and middle finger (Fig. 3b). The index finger guides and provides the downward force while the thumb and middle finger support the $200 \mu \mathrm{L}$ pipette tip.

10. The following steps will be described from the perspective via stereo microscope with $40 \times$ magnification. The prime directive is to extract embryo from each seed one-by-one through visual confirmation.

11. Disregard the orientation of the seed, position the tip at the 4-5 o'clock position at the proximity of the seed (see Note 19).

12. Apply downward force on the tip until seeing the seed deforms (getting bigger, pressed) ( $\mathrm{see}$ Note 20).

13. Maintain the same downward force and slide the tip toward the 10 o'clock position to the edge of the seed. An expulsion of materials from the seed, including an embryo, would be expected indicating successful embryo extraction (Fig. 3d). If no expulsion occurs, repeat twice without applying more 


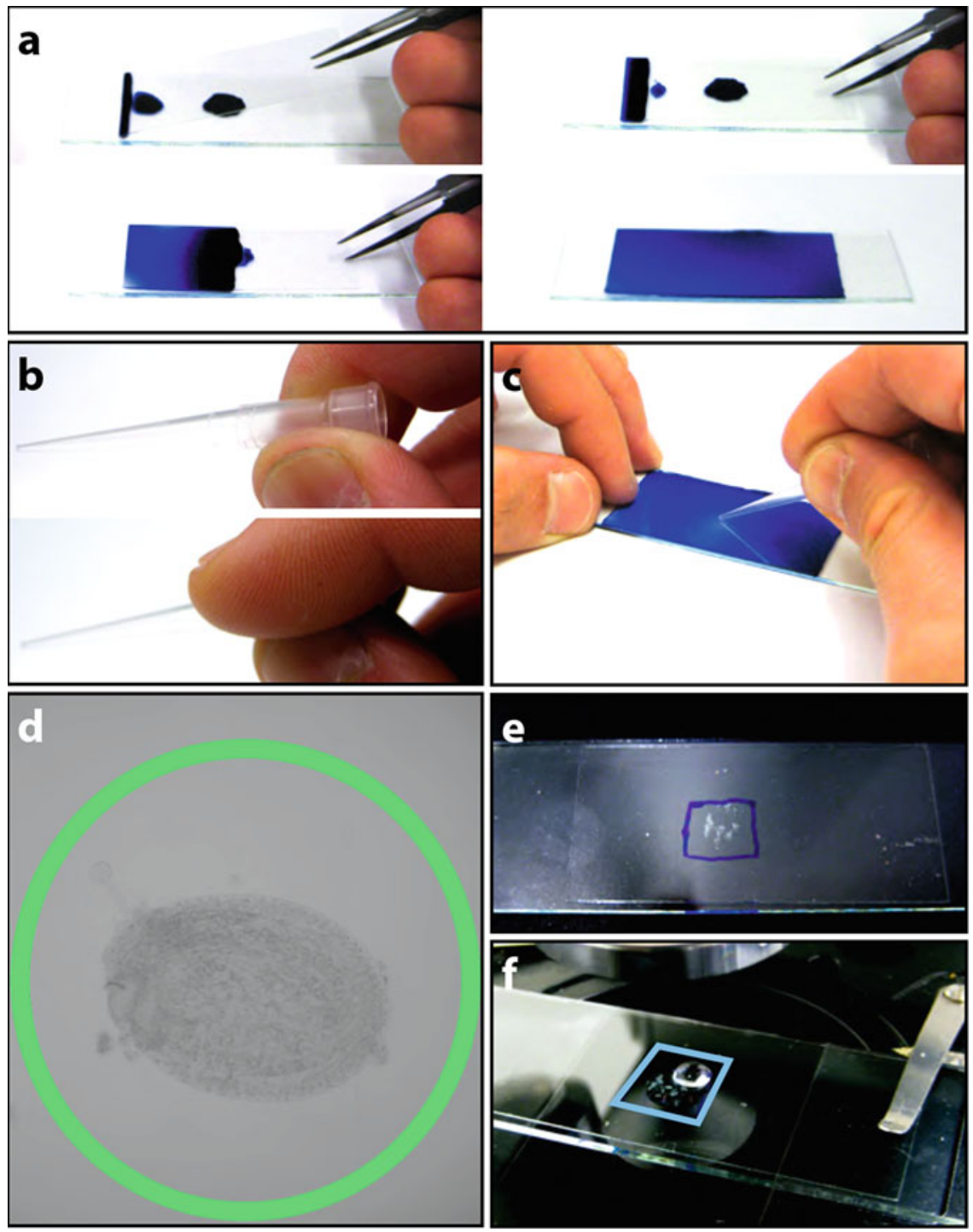

Fig. 3 Specimen preparation and embryo extraction. (a) Position of the staining medium (left side of the specimen) and placement of the coverslip. (b) Holding the pipette tip. (c) Hands position during embryo extraction. Note the position of the thumb, index, and middle finger of the left hand. (d) Seed with extracted embryo. The green area is the suitable area to apply pressure by the pipette tip during embryo extraction. (e) The imaging area marked by the permanent marker in the backside of the specimen. (f) Mounting the specimen on the microscope stage. Note the stage clip is on the coverslip and the relative position between the immersion medium and the marked imaging area (light blue quadrilateral). The operation of the objective lens must be remaining inside the marked imaging area

downward force than in the first attempt, but change the position and orientation of the slide, for example, change from 4-to-10-o'clock to 5-to-11-o'clock. If an expulsion occurs but with no embryo in the expelled materials or no expulsion after three attempts, move on to the next seed (see Note 21). 


\subsection{Imaging the Subcellular Structure Using an Upright Confocal Microscope}

14. Repeat Subheading 3.2, steps $\mathbf{1 2}$ and $\mathbf{1 3}$ till all seeds are processed.

15. Turn the specimen to vertical position so the coverslip is facing away from the operator.

16. Identify the position of the seeds and mark this area with permanent marker on the slide (the coverslip remains facing away from the operator) (Fig. 3e) (see Note 22).

1. Mount the specimen on the specimen stage of the microscope. Lift the working surface of the stage clip, position it above the left or right edge of the coverslip, and gently lower it to secure the coverslip (Fig. 3f) (see Note 23).

2. Add immersion medium on one of the seeds close to the mark (Fig. 3f) and raise the stage till the immersion medium contacts the objective lens.

3 . Find the embryo and acquire images as ordinary imaging procedure with following recommendation (see Note 24).

4. Use photon counting mode: The combination of live sample and repetitive scanning to generate optical stacks necessitates low laser input to prevent phototoxicity and photobleaching while the minute nature of subcellular structure and weaker promoter activity (while pWOX2 is considered strong embryo promoter, it is weaker than the commonly used p35S and pUBQ10 in more developed organs, such as roots) generates small quantities of photons per pixel. Therefore, photon counting is the preferred detector operation mode. Setting a discriminator equal to the background (an area without biological material), immensely increase image contrast and reduces background noise.

\section{Notes}

1. The difference between the blade for shaving and for industrial use is their sharpness and thickness. The sharper and thinner nature of razor for shaving make it ideal for cutting the silique valves open without damaging the seeds.

2. This orientation allows the operator to see the exact position of the point of the needle as the actual point of the needle will be at the left-hand side when the tip is pointing downward perpendicularly.

3. Essential for collecting seeds containing 1-16 cell embryo. Smaller tip dimension such as $0.05 \times 0.01 \mathrm{~mm}$ or $0.025 \times 0.005 \mathrm{~mm}$ will be preferred for seeds with developmental defect or containing $1-4$ cell embryos. 
4. We ordered $5 \mathrm{mg}$ Taxol and add $585 \mu \mathrm{L}$ of $100 \%$ DMSO directly to the stock vial with a $1000 \mu \mathrm{L}$ pipette tip whose first $5 \mathrm{~mm}$ point was cut off to accommodate the viscosity of $100 \%$ DMSO. Gently pipet up and down ten times for mixing. Alternatively, 50\% DMSO dissolved in demineralized water can be used.

5. This ensures firm valves and facilitates dissecting the siliques as firmer valves offer stronger mechanical feedback when cut by razor blade. Watering the plant a day in advance also ensures that seeds are turgid and increases the success rate of embryo extraction.

6. For wild types or mutants without a seed abortion phenotype, one silique is generally sufficient for each specimen. Otherwise, no more than 50 seeds per specimen should be used. This ensures that seeds are well separated from each other and provides sufficient room not occupied by the seed to be operated by the pipette tip during embryo extraction.

7. The light from beneath makes the silique, especially the younger siliques containing seeds with embryos younger than heart stage, transparent, making each seed in the silique more distinguishable so that the operator can evade the seeds when necessary.

8. Keep the fingers in one half of the razor blade to maintain clear view in stereomicroscope. Make the cuts $\sim 0.5 \mathrm{~mm}$ from both ends of the valves. This may eliminate $2-4$ seeds, but will make it easier to open the valves as these cuts remove the more tapered parts of the silique.

9. With the wrist and forearm rest on the table provides a stable platform for the finger movement. Try different path of the razor blade (with the Petri Dish and silique in the corresponding orientation), for example 9-3 o'clock, 11-5 o'clock, or 12-6 o'clock, to find the most stable movement for each operator. The safety razor blade is so sharp that as long as one feels the contact between the razor blade with the valve, the blade is making the cut already. If not, replace the razor blade.

10. These volumes are guidelines as the combined total volume of mounting and staining medium depends on the size of the seed, which is correlated to embryo's developmental stage in wild type plants. This step requires $1 / 3$ of the combined total volume of mounting and staining medium. Two criteria of the optimal combined total volume of mounting and staining medium are: firstly, sufficient liquid between the coverslip and the slide so no bubble occurs during embryo extraction; secondly, no liquid flowing outside the coverslip after embryo extraction. 
11. Start from the stigma end of the right valve as the funiculi of the seeds in the right valve is most accessible with this orientation of working. For seeds containing heart to torpedo stage embryos, $0.6 \times 15 \mathrm{~mm}$ needle is preferable with larger working surface to accommodate larger seeds.

12. Do not dip the whole tip of the needle/forceps into the droplet as this may encapsulate the seed within a small droplet on the tip instead of transfer the seeds to the mounting medium. Keep the needle/forceps dry as seeds are prone to stick to wet surface and this will only make it difficult to transfer the seeds to the mounting medium.

13. Similar to Subheading 3.1, step 13, these numbers are a guideline, but shall be $1 / 6$ of the optimal combined total volume of mounting and staining medium.

14. The lid prevents the minute volume of the mounting medium being evaporated causing change in the osmotic pressure of the mounting medium. The incubation makes the seed easier to be extracted; reducing the force required to push the embryo out of the seed and allows Taxol to enter the seed to stabilize microtubule.

15. Similar to Subheading 3.1, step 13, these numbers are a guideline, but shall be $1 / 2$ of the optimal combined total volume of mounting and staining medium.

16. Surgical gloves also increase friction between the finger and the glass and facilitate to secure the specimen.

17. It is crucial to have no air bubble as the air bubble will become an air cushion between the coverslip and the slide that hinders embryo extraction. In addition, the movement of the bubble could cause movement of extracted embryos during imaging. There will be some room left at the right edge of the coverslip that is not contacting the slide. This is intentional and will be sealed in the following steps.

18. The thumb and the middle finger provide support for the whole specimen. The index finger provides the support to secure the coverslip to prevent the movement of the coverslip during the popping. This is crucial as any horizontal coverslip movement will damage the seed and make it impossible to extract the embryo from the seed. Maintain left index finger position supporting the coverslip until the end of embryo extraction.

19. Never position the tip directly above the seed as the following pressure will likely squash the seed but not "pop" the seed.

20. Pay attention to the seed, especially those containing an embryo from globular or later stages. The seed could burst at this point if an expulsion of materials from the seed, including an embryo, is observed. 
21. Attempting to extract embryos from damaged seeds is futile because it is impossible to build up sufficient pressure in the seed to expel the embryo. The expected efficiency of embryo extraction is between $25 \%$ and $50 \%$.

22. This marks the area for objective lens operation. Each side of this marked area must be at least $8 \mathrm{~mm}$, or the diameter of immersion medium when the objective lens is at its operating position against the specimen, away from the edge of the coverslip. This mark serves as a landmark when searching for the embryos and prevents the objective lens being too close to the edge of the coverslip causing the contact/mixture of the immersion medium with the mounting medium that could drastically alter the property, such as osmotic pressure, of the environment surrounding the extracted embryo.

23. This prevents any horizontal movement of the coverslip that could damage the embryos.

24. Always take optical stacks: The isodiametric geometry of the embryo makes 3D reconstruction necessary for the analysis of subcellular organization.

\section{References}

1. Wendrich JR, Weijers D (2013) The Arabidopsis embryo as a miniature morphogenesis model. New Phytol 199:14-25

2. Llavata-Peris C, Lokerse A, Möller B, De Rybel B, Weijers D (2013) Methods Mol Biol 959:137-148

3. Musielak TJ, Schenkel L, Kolb M, Henschen A, Bayer M (2015) A simple and versatile cell wall staining protocol to study plant reproduction. Plant Reprod 28:161-169. https://doi.org/ 10.1007/s00497-015-0267-1

4. Yoshida $S$ et al (2014) Genetic control of plant development by overriding a geometric division rule. Dev Cell 29:75-87. https://doi. org/10.1016/j.devcel.2014.02.002

5. Benfey PN, Ren L, Chua NH (1990) Tissuespecific expression from CaMV $35 \mathrm{~S}$ enhancer subdomains in early stages of plant development. EMBO J 9:1677-1684

6. Völker A, Stierhof YD, Jürgens G (2001) Cell cycle-independent expression of the Arabidopsis cytokinesis-specific syntaxin KNOLLE results in mistargeting to the membrane and is not sufficient for cytokinesis. J Cell Sci 114:3001-3012
7. Sun CW, Callis J (1997) Independent modulation of Arabidopsis thaliana polyubiquitin mRNAs in different organs and in response to environmental changes. Plant J 11:1017-1027. https://doi.org/10.1046/j. 1365-313X.1997.11051017.x

8. Norris SR, Meyer SE, Callis J (1993) The intron of Arabidopsis thaliana polyubiquitin genes is conserved in location and is a quantitative determinant of chimeric gene expression. Plant Mol Biol 21:895-906. https://doi.org/ $10.1007 / \mathrm{BF} 00027120$

9. Gooh K et al (2015) Live-cell imaging and optical manipulation of Arabidopsis early embryogenesis. Dev Cell 34:242-251. https://doi.org/10.1016/j.devcel.2015.06. 008

10. Sauer M, Friml J (2008) In vitro culture of Arabidopsis embryos. Methods Mol Biol 427:71-76

11. Liao CY, Weijers D (2018) A toolkit for studying cellular reorganization during early embryogenesis in Arabidopsis thaliana. Plant J 93:963-976. https://doi.org/10.1111/tpj. 13841 